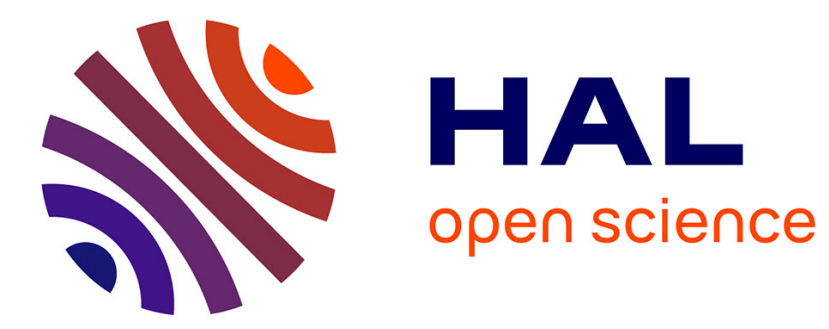

\title{
L'ÎLE ET LE TERTRE : ESSAI DE TOPOLOGIE LITTÉRAIRE
}

Paul Carmignani

\section{To cite this version:}

Paul Carmignani. L'ÎLE ET LE TERTRE : ESSAI DE TOPOLOGIE LITTÉRAIRE. Les lieux de la vie américaine, AFEA, May 1987, Baume-les-Aix, France. pp.294-301. hal-01742980

\section{HAL Id: hal-01742980 \\ https://hal.science/hal-01742980}

Submitted on 26 Mar 2018

HAL is a multi-disciplinary open access archive for the deposit and dissemination of scientific research documents, whether they are published or not. The documents may come from teaching and research institutions in France or abroad, or from public or private research centers.
L'archive ouverte pluridisciplinaire HAL, est destinée au dépôt et à la diffusion de documents scientifiques de niveau recherche, publiés ou non, émanant des établissements d'enseignement et de recherche français ou étrangers, des laboratoires publics ou privés. 


\title{
L'ÎLE ET LE TERTRE : \\ ESSAI DE TOPOLOGIE LITTÉRAIRE
}

\author{
P. CARMIGNANI \\ Université P. Valéry-Montpellier III
}

L'ORIGINE DE CET ESSAI sur deux pôles particuliers de la topographie culturelle
et littéraire du Nouveau Monde, figurent deux lieux communs de la critique
des grands classiques américains. Le premier, formulé par D. H. Lawrence (Studies in Classical American Literature, 1922), pose comme principe que l'âme ou le génie des lieux a donné son inflexion particulière à la voix qui s'exprime dans le roman américain ; le second, énoncé par L. Fiedler (The Return of the Vanishing American, 1968), met l'accent sur le fait que la littérature américaine s'est, plus que toute autre, définie par rapport à l'espace, comme en témoigne l'émergence d'un genre propre à l'Ouest - le Western - ou même d'un parler - le tall tale - à la mesure de la « spaciosité de la République ».

Ces propositions, bien que très générales, ouvrent la voie à une topoanalyse dégageant la valeur déterminante des lieux, non pas fictifs mais bien réels, où l'imaginaire se réalise et s'ancre la fiction. Dans La Terre et les rêveries du repos, G. Bachelard écrit qu'il existe des «images conteuses », c'est-à-dire des images produisant automatiquement un conte parce qu'elles demandent qu'on imagine un avant et un après. Pour ma part, je soutiens qu'il existe également des «lieux conteurs » qui sont, comme les quatre éléments matériels, opérateurs d'images et inducteurs de rêveries mythopoétiques (cf. S. Ann Grau, The Keepers of the House : «Every place, every person has a ring of stories around them, like a halo almost», 14 ). Mainte œuvre témoigne de l'enracinement de la fiction dans un lieu particulier et sur le cadastre des fictions américaines figurent en qualité de «lieux-dits » (entendez de topoï sur lesquels s'articule un logos), la Prairie, la Forêt, le Fleuve, mais aussi la Route - chère à J. Kerouac -, la Plantation, la Mine ou la Ville. Dans cet espace matériel mais aussi graphique et symbolique, j'ai choisi deux sites - l'île et le tertre - qui sont, comme la suite le montrera, liés par un certain rapport de symétrie et de complémentarité.

L'île est un des lieux clés de la littérature américaine et, de l'île au trésor, Sullivan's Island, qui sert de cadre à “The Gold Bug”, à l'île antarctique de Tsalal où Arthur Gordon Pym accomplit son voyage au bout de la nuit, en passant par les îles exotiques de Herman Melville, 
comme Typee, l'Éden érotique mais hélas cannibale des Mers du Sud, les exemples célèbres sont légion. C'est la raison pour laquelle je restreindrai mon domaine d'étude au type d'île qui est sans conteste le plus authentiquement américain, c'est-à-dire non pas l'île marine mais l'île fluviale au milieu du Mississippi, qui représente, plus encore que l'Océan, la frontière naturelle et mythique entre l'Est et l'Ouest, l'Ancien et le Nouveau Monde. L'exemple classique en est évidemment Jackson's Island, l'île-refuge de Huckleberry Finn et de son compagnon, Jim, l'esclave fugitif. C'est là que pour la première fois, sous la plume de Mark Twain et dans une langue vernaculaire, se met en place un des mythes fondamentaux de la fiction américaine : la cavale, la fugue loin de la société et de la civilisation, la tentative de retour vers un espace édénique où l'adolescent blanc et le grand enfant noir peuvent réaliser - le temps d'une brève parenthèse -, l'idéal de la pastorale et de la fraternité entre les hommes. On sait que par la suite, obligés de fuir ce havre de paix, Huck et Jim descendent le fleuve sur un radeau, évident substitut de l'île, et qu'à partir de là, le récit se déroule au rythme d'une alternance symbolique entre la dérive idyllique au fil du Mississippi et les dangereuses escapades sur les berges où se trouve le monde cynique et cruel des adultes.

Avec le roman de M. Twain, les grandes lignes du mythe de l'île fluviale édénique sont fixées ; l'archétype connaîtra de multiples variantes et sera même parodié - preuve de l'existence d'un modèle canonique - comme c'est le cas dans une nouvelle d'Erskine Caldwell, intitulée "Maud Island" (Men and Women, New York, Signet Classic, 1962). Sur l'île, bornée à l'ouest par le Mississippi et à l'est par un marécage, abîme de perdition jouxtant le Paradis, trois personnages ont établi leur campement. Mais l'harmonie régnant entre Uncle Marvin, miprédicateur, mi-fermier, et les deux adolescents, Jim et Milton, est perturbée par l'arrivée inopinée dans ce lieu jusqu'alors vierge de femmes, d'un plaisancier et de deux séduisantes créatures. Malgré les rebuffades d'Uncle Marvin, les étrangers accostent sur lîle ; le diable est dans la place et le serviteur de Dieu succombera aux charmes de ces deux jeunes pécheresses. Il plie armes et bagages, rembarque les enfants, médusés, et revient goûter seul aux délices du fruit défendu.

Ces deux exemples constituent une toile de fond qui fera ressortir avec plus de relief la manière dont le thème de l'île a été repris et développé par l'écrivain sudiste contemporain, Shelby Foote, dans son roman Follow Me Down, publié en 1950. Les diverses potentialités du motif de l'île fluviale ont rarement été mieux explorées que dans cette œuvre exemplaire. L'intrigue, une fois réduite à sa plus simple expression, se présente comme suit : un petit fermier puritain, Luther Eustis, délaisse, à cinquante ans, araire et foyer pour nouer une liaison. avec Beulah Ross, une fille de joie de dix-huit ans. L'idylle, qui a pour cadre une l'île où Luther 
s'est rendu, lorsqu'il était enfant, se dénoue tragiquement au bout de deux semaines : Luther étrangle Beulah, la jette dans le fleuve, et s'en revient, tranquille comme Baptiste, vivre entre les siens le reste de son âge. Ce Redneck's Progress met en lumière les dimensions allégoriques, mythiques et mystiques de l'île explicitement assimilée au «paradise before Eve and the snake corrupted it », (116); de même, les deux protagonistes ont le sentiment de rejouer un scénario bien connu : celui de « old Adam and Eve » (117).

Pour rejoindre l'île, il faut traverser une partie du Mississippi et ce franchissement prend l'allure d'un véritable rite de passage ; en effet, le fleuve est une frontière et l'île se trouve au cœur d'une triple opposition d'ordre spatial, temporel et spirituel.

Oasis de verdure et de paix, l'île est une sorte de lieu hors cadastre représentant l'antithèse de la société : c'est le lieu du dépouillement, de la nudité, de la rupture avec l'ordre et les rythmes ordinaires. À ce titre, elle s'oppose au foyer (home), monde du quotidien et du labeur, de la contrainte et de la norme.

L'île est non seulement hors de l'espace quotidien, elle est aussi hors du temps présent. L'île vierge symbolise un monde prélapsaire, l'âge d'or de l'humanité. En retournant sur l'île, Luther Eustis effectue une remontée dans le temps, qui devrait en principe le rapprocher de la pureté originelle et de l'innocence première. Lieu du renouveau et de l'éternel recommencement, lîle jetterait un pont entre un hic et nunc prosaïque et un illud tempus mythique.

Enfin, l'île symbolise une opposition fondamentale entre deux sphères représentées dans le récit par l'antithèse "this world and that world", c'est-à-dire l'ordre profane et l'ordre sacré. Site mystique, l'île est promesse d'extase ; elle doit servir de cadre aux noces de Beulah dont le nom biblique signifie «la Promise » ou «la Terre Promise » et Eustis, étymologiquement, «le bel épi ». Il y a bien subversion de l'ordre profane, mais l'ouverture sur le religieux ou le sacré ne se réalise qu'au prix de la violence ; lîle devient le lieu où se produisent deux transgressions de la vie régulière et ordonnée en société, l'orgasme et le crime, l'amour et la mort. Mais à la suite du crime perpétué par Luther Eustis, la Terre est souillée, le Paradis perdu et le Pacte rompu. L'Éden est corrompu, transformé en terre gaste ; la magie s'évanouit et l'île perd tout son charme : elle paraît plus petite, son sable est sale, ses arbres rabougris et desséchés ; alors qu'on la croyait vierge, elle est occupée par des grotesques (Miss Pitts, une hommasse moustachue et son fils Dummy, un sourd-muet disgracieux) ; enfin, elle a été investie par des forces maléfiques en la personne d'un sectateur du culte vaudou, qui a en plus le mauvais œil. Ainsi, les connotations positives d'abord conférées à l'île sont dans un deuxième temps changées en leurs contraires ; loin de représenter une Arcadie insulaire, lîle, lieu de la 
transgression et de l'impossible séjour, est le théâtre d'un drame où Luther Eustis, l'élu à rebours, et Beulah Ross, pitoyable Lilith, rejouent l'épisode le plus tragique du scénario biblique qui sous-tend cette histoire, à savoir la Chute et la perte de l'Éden.

Ces divers exemples démontrent, même a contrario, qu'il existe une étrange affinité entre le paysage américain et l'idéal de la pastorale, mais que le lieu le plus propice aux songeries sur l'origine et l'idéal bucolique, c'est l'île verdoyante. L'île symbolise l'Amérique telle que la rêvait l'imaginaire européen ; d'ailleurs, dans l'histoire, même de la découverte et de la conquête du Nouveau Monde, c'est l'île qui est première et non le continent. La genèse de l'Amérique se fait à partir de l'île, qu'il s'agisse de San Salvador, où C. Colomb prit pied, le 12 octobre 1492, ou de Roanoke Island, le premier avant-poste de la colonisation. La littérature et l'imaginaire témoignent de la même antériorité ; par exemple, dans The Great Gatsby, Nick Carraway évoque :

«the old island here that flowered once for Dutch sailors'eyes - a fresh, green brest of the new world. [...] For a transitory enchanted moment man must have held his breath in the presence of this continent, compelled into an aesthetic contemplation he neither understood nor desired, face to face for the last time in history with something cornmensurate to his capacity for wonder. » (222-223).

C'est également de Long Island, l'île en «forme de poisson », au vieux nom indien oublié de «Paumanok » que part Walt Whitrnan, le premier aborigène blanc, pour composer son «chant pour un Nouveau Monde », et c'est enfin de Jackson's Island que s'élève la première voix authentiquement américaine.

En fin de compte, le continent et l'île ne relèvent ni du même désir ni du même imaginaire ; le continent, favorisant l'extraversion, le dynamisme et les « rêveries de volonté », donnera naissance au mythe de l'Ouest et de la Frontière ; l'île fluviale - lieu de l'intimité et du refuge -, est placée sous le signe de l'introversion et du psychisme involutif ; elle est liée aux rêveries du « repos enraciné » et au mythe du retour aux origines.

Le second topos de mon diptyque spatial, c'est le tertre indien, élévation de terre servant de mausolée à certaines tribus du bassin du Mississippi et du sud est des États-Unis.

Le tertre est semblable à une l'île émergeant des terres ; telle une épave abandonnée dans l'espace par le reflux du temps, il domine une Prairie souvent assimilée à un Océan. Les pionniers la sillonnaient à bord de Prairie schooners (littéralement «goélettes »), et les émules de J. Kerouac, l'écumeur des terres, la traversent au volant de voitures qui, nous dit l'auteur, « hold the road like a boat holds on water» (On the Road, 216). 
Vestige de la véritable Amérique, archaïque et originelle, le tertre rappelle l'instant déterminant de l'histoire du Nouveau Monde : la rencontre de l'Indien avec l'envahisseur blanc. Dans tous les récits où il se manifeste, comme une image fugitive ou une présence obsédante, que ce soit Requiem for a Nun de W. Faulkner, The Keepers of the House de S. Ann Grau, A Time and a Place de W. Humphrey ou September September de S. Foote, le tertre îlot du souvenir émergeant du «grand marécage blanc»-, évoque le fantôme inapaisé de l'aborigène, hantant un paysage arnéricain «qui n'a jamais été en accord avec l'homme blanc » (H. Lawrence). Affleurement du passé, le tertre est à la fois archive matérielle du temps passé et registre où s'inscrivent en termes de passif les injustices commises par l'Européen. Le point de vue qu'il offre sur l'histoire collective est celui de l'autochtone et à ce titre, il représente sur le cadastre des fictions américaines une position exceptionnelle. Pour en parler, je recourrai à deux nouvelles, extraites de l'œuvre de W. Humphrey précédemment citée, et du-cinquième roman de S. Foote, Jordan County (1954), qui est à la fois exploration d'un espace (le Delta) et remontée dans le temps.

Jordan County est un lieu dit mais aussi le «dit» d'un lieu ; c'est un roman-paysage ou un paysage romancé à travers lequel se déroule une quête : celle des origines et de la vérité. La septième et dernière nouvelle de ce recueil, "Le Tertre sacré", marque le point d'aboutissement du pèlerinage à rebours du temps que retrace Jordan County. On atteint ainsi, paradoxalement, la limite extrême et le cœur de cette investigation d'un passé, qui, comme « toute interrogation sudiste sur l'histoire, suppose presque un attendu métaphysique : quelle faute a valu tel châtiment? »(M. Gresset, 119). La réponse figure dans un procès-verbal où le greffier Andrew Benito Courbiere consigne la déposition de l'Indien, Chisahahoma, venu s'accuser d'avoir participé sur le tertre sacré, par une nuit de pleine lune, au sacrifice de deux trappeurs blancs.

Le témoignage de l'Indien évoque une longue période, de 1797 à 1540, et décrit du point de vue du colonisé, l'histoire du Sud depuis l'exploration du Mississippi par Hernando de Soto jusqu'à l'annexion de cette province par les États-Unis. Ces deux événements constituent le prologue et l'épilogue d'une tragédie qui s'est déroulée en quatre actes - conquête militaire, évangélisation, exploitation économique et consécration juridique de la spoliation des vaincus - et a transformé l'Éden primitif en Enfer civilisé.

"Le Tertre sacré" évoque un sacrifice et un sacrilège, c'est-à-dire l'intrusion d'un élément étranger et perturbateur dans un milieu dont l'équilibre et la paix dépendaient de son maintien à distance, de l'autre côté de l'Atlantique. L'harmonie régnant entre l'aborigène et le 
continent américain est définitivement rompue quand entre en scène l'Européen et avec lui, le Mal.

Entre l'Indien et l'Européen, il n'est point de communion possible; le commerce en tient lieu et c'est ainsi que s'organise un monstrueux marché de dupes au cours duquel le PeauRouge troquera son Dieu, Nanih Waiya, contre le Crucifié, son or contre du toc et sa terre contre de l'argent ; il recevra en prime la civilisation et la vérole pour solde de tout compte. Les deux victimes en sont porteuses et la tribu contaminée est vite décimée par ce terrible fléau. Pour apaiser les dieux, le chef délègue Chisahahoma, l'aède, comme victime expiatoire auprès des autorités espagnoles de la province, mais son envoyé, après s'être converti à la religion de l'occupant, est, lors du procès, lavé de tout soupçon et renvoyé chez les siens porter la bonne parole. Comble de l'ironie, le Peau Rouge est blanchi... Ce qui ressort du témoignage de l'Indien, c'est que la spoliation des terres constitue la principale forfaiture des Blancs :

Then, lo, they began to ask a strange thing of us, seeking to buy the land. Sell us the land, they said : Sell us the land. And we told them, disguising our horror : No man owns the land; take it and live on it ; it is lent you for your lifetime ; are we not brothers ? (287)

Il n'est pour l'Indien pire sacrilège ; la terre est un dépôt sacré, un bien communautaire indivisible et inaliénable, mais le Blanc va parceller et morceler l'espace vierge et transformer ainsi la matrice tellurique en matrice cadastrale. Le Mal est fait ; l'Indien et le Blanc deviennent des frères ennemis : Caïn, l'Européen, le cultivateur du sol, l'emporte sur Abel, l'autochtone nomade. Dès lors, l'Amérique entre dans l'histoire, ou plutôt y choit, et c'en est fait, de l'innocence première et de la tendre pastorale.

Ce que le tertre rappelle, c'est que pour faire de l'Amérique un Paradis retrouvé, il fallait que le vieil Adam européen célébrât de nouvelles noces - non pas avec une femme, fûtelle prénommée, Beulah, la Promise, ou Columbia, la déesse néoclassique de la liberté - mais, avec celui qui incarnait le nouvel Adam et l'authentique Américain, c'est-à-dire l'Indien, le Peau-Rouge.

L'île dans Follow Me Down et le tertre dans Jordan County représentent deux variantes antithétiques du mythe du Paradis terrestre ; lîle fluviale est un Éden chrétien, biblique, où, mû par l'illusion que la femme, qui nous a valu le péché peut aussi nous ouvrir l'ère du salut, Luther Eustis cherche paradoxalement à regagner le Paradis grâce à celle-là même qui en a fait chasser l'homme.

Le tertre, symbole d'une autre promesse, incarne un Éden païen et sauvage, qui disparaît sans espoir de retour avec l'avènement du Christianisme et l'intrusion de la femme dans 
un monde vierge. Il est donc associé à ce que L Fiedler appelle «le Mythe des gais compagnons dans les terres vierges et sauvages », c'est-à-dire :

the Myth of the Good Companions in the Wilderness, the story of a White Man and a Red who find solace and sustenance in each other's love, identified for most Americans with the encounter of Fenimore Cooper's Natty Bumpo and Chingachgook over the embers of a dying fire (51).

La seconde nouvelle - "The Last of the Caddoes" - de W. Humphrey, confirme cette interprétation et apporte en outre la preuve qu'il existe bien au fond, selon la thèse de L Fiedler :

two archetypal versions of the Fall in the Garden : one inherited from the Old World, one created in the New. In the first, Man and Women are portayed living at peace in an Earthy Paradise until the Serpent enters ; in the second, Man and the Serpent are presented as cohabiting amicably until Wornan comes on the scene (116).

Dans cette nouvelle, un adolescent, Jimmy Jawkins, apprend par hasard, au cours d'une dispute avec sa mère, qu'un peu de sang indien coule dans ses veines. C'est une révélation qui bouleverse sa vie et, pour recueillir l'héritage que tous les siens ont dédaigné, il entreprend de fouiller le tertre qui se dresse au milieu du champ de coton de son grand-père paternel, un métis renégat :

Commanded by the voice of his people to know himself through knowing them, Jimmy had bared the buried history of the Caddoes, delving backwards in time from their end to their beginning (193).

La célébration du treizième anniversaire de Jimmy est l'occasion d'une nouvelle dispute au cours de laquelle sa mère, venue visiter le tertre, l'accuse d'être «snake in your mother's bosom » (201). L'adolescent, comprenant que ses ancêtres lui parlent par la bouche de sa mère, fait de cet anathème une sorte de nom secret. Il l'adopte, le revendique, et cet adoubement symbolique rétablit la continuité du lien tribal et replace l'adolescent dans la lignée dont il était exclu. Le conflit mère/fils s'exacerbe jusqu'au jour où Jimmy quitte le foyer pour se réfugier à l'intérieur du tertre et là, survient une épiphanie décisive. un vieux serpent noir mue sous les yeux de l'adolescent et lui laisse sa peau en signe d'une nouvelle alliance. Mais cette confrontation, qui tient du rite de passage et d'initiation, est interrompue par l'intrusion de la mère, venue reprendre ce fils rebelle qui finira, dit-elle, par la faire mourir. La fin du récit mérite d'être citée in extenso :

In obedience to his victim's nod, Snake-in-his-Mother's-Bosom took up his suitcase and followed her down the steps and across the barren field to the car. In its reaview mirror he watched the mound diminish and finally disappear. The snakeskin rode on his lap. Now he must wait. Must wait for their next, their final command. It would not come soon ; they sipped 
their pleasures slowly. Many times yet he would have to hear his mother say that he would be the death of her. So many times that when the final order came it would be almost welcome, a release. Distant and ghostly, it sounded already in the echoing silence of his mind. Over and over, like a phonograph record when the needle cannot find the starting groove. «Kill me at once then and be done with it!» his mother's voice was saying. One day the needle would find the groove. Then out would come the command loud and clear and with the sudden shock of long-expectedness. Then Snake-in-his-Mother's-Bosom would strike, accomplish his mission and fulfill the prophecy ; and then at last the ghost of the Caddoes could lie down at peace in their many-tiered mound and haunt the land and him no more.

Le rêve éveillé de l'adolescent s'oppose à la représentation chrétienne de la ViergeMère écrasant sous son talon la tête du serpent maudit ; cette fois-ci, le serpent prendra sa revanche et éliminera la rivale lui disputant le cœur de Jimmy.

Que conclure au terme de cette brève incursion dans un espace physique mais aussi graphique et mental? Deux points essentiels ; en premier lieu, que la fiction du Nouveau Monde manifeste la présence d'un symbolisme et d'une mythologie fondés sur l'espace, et ensuite, qu'elle démontre l'existence d'une « géographie morale de l'imaginaire américain » (Leo Marx, The Machine in the Garden, 72). Un paysage peut ainsi devenir le support et le prétexte d'une fable morale ; dans Follow Me Down et "The Last of the Caddoes" la topique débouche sur une métaphysique (la question du Mal et de la Culpabilité) et l'archéologie, sur une téléologie : vers quelle fin tend l'histoire du Nouveau Monde ? Est-elle en passe d'accomplir la vision exaltante d'un Thomas Paine, déclarant à l'aube de l'histoire américaine :

We have it in our power to begin the world. over again. A situation, similar to the present, hath not happened since the days of Noah until now. The birthday of a new world is at hand, and a new race of men, perhaps as numerous as all Europe contains, are to receive their portion of freedom from the events of a few months.

L'île du Mississippi et le tertre indien sont les lieux d'une célébration et d'une démythification de la pastorale américaine ; ils révèlent qu'au cœur du passé américain, figure non pas une innocence première mais une culpabilité originelle et rappellent comment le Nouveau Monde, terre promise, Paradis géographique, a été mué en enfer par l'histoire.

Si l'espace est bien une sorte d'inconscient des civilisations, alors l'île fluviale - lieu du fantasme et du désir (retour vers l'Éden, transgression...) - et le tertre - lieu du refoulé de l'histoire collective (négation de l'Aborigène, forclusion de la sauvagerie) - sont deux topö̈ essentiels de la littérature américaine, car ils mettent en lumière la dialectique fondamentale de l'innocence et de la culpabilité, et révèlent enfin que le mythe édénique - s'il n'a pu trouver place dans le domaine des faits -, a trouvé refuge dans celui de la fable et de la fiction. 


\section{INDEX DES OEUVES CITÉES}

- G. BACHELARD. La Terre et les rêveries du repos, Paris, J. Corti, 1948.

- E. CALDWELL. "Maud Island" in Men and Women, New York, New American Library, 1962.

- D. H. LAWRENCE. Studies in Classical American Literature, Harmondsworth, Penguin Books, 1978.

- L. FIEDLER. The Return of the Vanishing American, New York, Stein \& Day, 1968.

- F. S. FITZGERALD. The Great Gatsby, Harmondsworth, Penguin Books, 1974.

- S. FOOTE. Follow Me Down in Three Novels, New York, Dial Press, 1964.

“The Sacred Mound" in Jordan County, publié dans Three Novels.

- S. A. GRAU. The Keepers of the House, New York, A. Knopf, 1969.

- M. GRESSET. "La Tyrannie du regard ou la relation absolue : Origine, émergence et persistance d'une problématique du mal dans l'œuvre de William Faulkner", Thèse, 1976, Vol. I.

- W. HUMPHREY. "The Last of the Caddoes" in A Time and a Place, New York, A. Knopf, 1968.

- J. KEROUAC. On the Road, Harmondsworth, Penguin Books, 1958.

- L. MARX. The Machine in the Garden, New York, OUP, 1967.

- The Works of Thomas Paine, New York, WMH Wise \& Company, 1934. 